\title{
Coal Mine Gas Early Warning System Based on Fuzzy Data Fusion Technology
}

\author{
Xiaoming $\mathrm{Wu}$, Chao $\mathrm{Xu}$, Yue $\mathrm{Ma}$ \\ Phone number:15055110896 1333919936815056932700
}

Emil address:2721822385@qq.com graymagpie@163.com 1278518415@qq.com

Zip code:230601

School:Anhui University

Address: No.111 Jiulong Road of Shushan District, Anhui University, Hefei, Anhui Province

Author's brief introduction: third-year student in Electronic Information Engineering College( Xaioming Wu, Yue Ma)

Chao Xu:Professor and Associate Dean, School of electronic and information engineering, Anhui University

Keywords: coal mine safety, fuzzy data fusion, gas warning

\begin{abstract}
The process of exploration and production in coal mines have many potential dangers so the coal mine safety problem has become the focus of social concern.Mine gas early warning system is an important measure that guarantee coal mine safety production because coal mine gas explosion is an important cause of coal mine accidents. In order to improve the accuracy and timeliness of the disaster early warning and reduce accidents,we research and develop the technology of coal mine gas early warning system based on fuzzy data fusion technology.

With the development of the time,the source of energy become diversified than before.But for now, primary energy still play a major role in the energy structure.Coal resources accounted for $67 \%$ of primary energy structure,more than the sum of other energies.The government expects the proportion of coal in primary energy consumption structure is still around 50\% until 2050 so the coal energy is the lifeblood of a country's economic development.But every year in China the number of deaths because of coalmine accidents has been very high, most of the accidents were caused by a gas explosion. Therefore, mine gas concentration monitoring and early warning are very necessary. Here, the author of this paper designed a coal gas warning system based on fuzzy data fusion technology.
\end{abstract}

\section{System overview}

In view of the existing problems in the coal mine gas monitoring system, we propose to design a kind of coal gas warning system based on embedded ARM, the Linux kernel transplantation and multi-sensor fuzzy data fusion technology. We collect information for downhole safety though the node multilayer cluster topology and control, by using temperature, wind speed, humidity, gas sensors and process, judge, summarize it. At the same time, the information of downhole parameters will be sent to the PC software system via mobile network. Then the PC will add data to a database of data entry and doing the secondary processing of data and giving some relevant parameters and forecast. People are involved in the system to set the corresponding data collection standards and the tertiary treatment is formed. Eventually the synthesis processing results will be integrated and displayed in the upper machine. With the character of real-time collection, real-time input, intelligent judgment and timely feedback, the system can process information multilevely and feedback it timely.

\section{The overall structure of the system and working principle}

The system is mainly composed of ground and underground monitoring substation, the main 
safety power supply, various sensors, frequency converter and the ventilator. Monitoring substation collect data from the sensor, after processing and judgment the data and generate the corresponding state display and alarming on the spot. At the same time, according to fusion result of normal, mild and dangerous these three monitoring substation, we can control the frequency converter output so as to control the fan speed. Firstly, monitoring substation send data to Ethernet by CAN bus. Secondly, CAN gateway access the optical fiber to Ethernet. Thirdly, Ethernet uses the ring network structure to ensure the security and reliability of data transmission. In the end, the master station in the ground analyze and process the data and display dynamic information such as text, curve.

2.1 master station on in the ground

The master station in the ground is constituted by the PC host, Ethernet switch RS232 module, printer and projector. Substation monitoring data switch into RS232 signals through the ground master and it will be sent to the PC host. Use the MSComm control to send and receive a serial port data through writing driver software by VB which is supported by DDE technology, then the main configuration communicate by DDE technology and VB so as to realize the function of upper machine data acquisition. Ethernet switch RS232 module using ETH232 GH Ethernet/serial converter. It is a photoelectric isolating Ethernet/serial converter and it isolateth the Ethernet, power supply and serial port.

2.2 monitoring substation underground

The monitoring substation includes S3C2440A main control chip, extended memory, signal acquisition module, LCD display, RS485 interface and CAN communication module, etc. For utilizing collected environmental information comprehensibly and improving substation alarm function, monitoring substation also introduces the fuzzy data fusion algorithm of multi-sensor which can not only make the fusion results more accurate and reliable but also control the speed of the fan according to the results of fusion. After the sensor information analysis and processing, monitoring substation use CAN bus and Ethernet gateway for communication.

\subsection{System for modular design}

As the coal mine underground environment is extremely sever and complex and different coal mine differs in thousands of ways, the project adopts modular structure design of software and hardware and each link is designed into the strong configurability minimum system on the premise of reliable. The flexible component system adapt to the requirements of the different size, different engineering environment installation, configuration, commissioning, etc. Modular design can not only shorten the design cycle, reduce cost, but also increase the quality of the product reliability. At the same time,it can easily transfer to forest fire prevention, underwater in the field of environmental monitoring, etc.

2.4 The fuzzy data fusion

Fuzzy data fusion is a process of multivariate information processing, firstly applied in the military field. As the further development of research and application, it gradually formed an independent discipline. Fuzzy data fusion analyze and integrate the information from multi-source or more sensors and data , eliminate redundant information and the possible conflict and get a description of the system consistency. This makes the system response faster and the conclusion is more accurate then it come to the final decision and estimate the tasks.

Most of the existing gas monitoring system use the bayesian bayesian estimation, D - S evidence theory. However, the coal mine gas accident usually happens suddenly and has no reliable prior probability which makes early warning not effective and timely. This project adopts the fuzzy set theory to avoid the prior probability. Also, the analysis and integration of data and information from multi-source or multi-sensor can not only avoid the limitations of single sensor and collect more comprehensive information but also deal with the uncertainty of the sensor information. Thus it can make the early warning function of the system more reliable and faster and the early warning information more accurate to some extent.

The process of fuzzy data fusion processing multi-sensor information:

(1)Signal acquisition: generally uses the special sensor then converts electrical signals to digital quantity that computer $\mathrm{I} / \mathrm{O}$ port can receive through $\mathrm{A} / \mathrm{D}$ transformation. 
(2)Data preprocessing: the main function is to improve the SNR of the signal, remove the noise and interference in the maximum degree. The methods are: averaging, filtering, removing outliers, etc.

(3)Feature extraction: feature extraction of the original information from the sensors. Features are various physical quantities that can be measured.

(4)Fusion calculation: analysis, choice andsupplementary of multi-sensor observations, generation of synthetic state and real time modifications.

\section{Conclusion}

Coal mining has a close relation with our country's economic development and social stability. In line with people-oriented and to strengthen the safety in production. we improved the gas early warning system. It realized higher accuracy and faster reaction. Modular design simplified the system design,saved the cost and the solution to local problems. This system reduced the risk of accident and ensured the safety of the lives of mining workers. It can be predict that the information of the environment dynamic monitoring and analysis will be widely applied in all areas of life and industrial production in the future. On the basis of this system, we can expand and modify it functionally. The application field of this kind of environment monitoring system must be wide and valuable.

\section{Reference}

[1]jingkui li.The coal mine gas monitoring system based on wireless sensor network research and design[J].Coal technology,2013,(11)

[2]shimin xu.Study of coal mine monitoring substation platform[J].Shandong coal technology,2016,(2)

[3]yong yan,xiangbing zhou.Based on the Internet of mine gas dynamic monitoring and early warning system design[J].Laboratory research and exploration,2016,(1) 